\title{
In-Vehicle Stress Monitoring Based on EEG Signal
}

\author{
${ }^{*}$ Shahina Begum, ${ }^{2}$ Shaibal Barua, ${ }^{3}$ Mobyen Uddin Ahmed \\ School of Innovation, Design and Engineering Mälardalen University, SE---721 23, Sweden \\ Corresponding Author: Shahina Begum
}

\begin{abstract}
In recent years, improved road safety by monitoring human factors i.e., stress, mental load, sleepiness, fatigue etc. of vehicle drivers has been addressed in a number of studies. Due to the individual variations and complex dynamic in-vehicle environment systems that can monitor such factors of a driver while driving is challenging. This paper presents a drivers' stress monitoring system based on electroencephalography (EEG) signals enabling individual---focused computational approach that can generate automatic decision. Here, a combination of different signal processing i.e., discrete wavelet transform, largest Lyapunov exponent (LLE) and modified covariance have been applied to extract key features from the EEG signals. Hybrid classification approach Fuzzy-CBR (case-based reasoning) is used for decision support. The study has focused on both long and short-term temporal assessment of EEG signals enabling monitoring in different time intervals. In short time interval, which requires complex computations, the classification accuracy using the proposed approach is $79 \%$ compare to a human expert. Accuracy of EEG in developing such system is also compared with other reference signals e.g., Electrocardiography (ECG), Finger temperature, Skin conductance, and Respiration. The results show that in decision making the system can handle individual variations and provides decision in each minute time interval.
\end{abstract}

Keywords: Stress, Monitoring System, Electroencephalography (EEG), Case-Based Reasoning (CBR), Largest Lyapunov Exponent (LLE)

\section{INTRODUCTION}

Monitoring vehicle drivers when they are exhausted with mental stress is an important feedback for a driver, especially to prevent accident, which could have large consequences both on lives and economical costs. Today, intelligent driver monitoring systems to assist drivers based on physiological sensor signals have drawn a lot of attention from the research community and industry. EEG signals contain important information about the mental state of the brain. EEG has widely been used, but not limited to, sleep study, epilepsy, neuroscience, and cognitive science $[1,2]$. EEG signals if processed correctly and efficiently have potential to facilitate advanced monitoring and diagnosis. EEG parameters and electrode positions correlated to different tasks and relax period are investigated in the paper [3]. The authors have suggested that 'stressed' and 'relaxed' states could be distinguished by a priori expected behavior (PEB) i.e., a significant difference between activity phases and rest period. The results of the study show that most of the PEB parameters are assembled at theta and alpha bands of the EEG signals. Also frontal channels mainly F3, Fz, F4 concentrate on these parameters. Paul D. Tyson[4] has investigated the alpha biofeedback for task related stress. The experimental results have supported the hypothesis that the high amplitude alpha is sensitive to the manipulation of contingent stress. In the paper [5], using the alpha symmetry method, effect of stress induced and binaural beats sounds on EEG alpha wave signal has been investigated. The authors have reported that for the stress induced cases alpha level was elevated in the right hemisphere of the brain than the left hemisphere. Also, the results have indicated that the alpha wave is significantly affected by the stressed induced and binaural beats sounds. In 2014, a study was done by Shamsul et al. [6], where the goal was to compare driving stress, fatigue and driving error between complex and monotonous driving. The authors have reported that the highest mean was observed in the theta waves compared to alpha and beta waves. Theta was higher in monotonous driving. Alpha waves mean was lowest for the monotonous and complex driving. Moreover, driving errors were measured using RORI (number of running-of-the road) and LSV (Large Speed variation). Both RORI and LAS were higher during complex driving. Further, stress level increased in the monotonous driving environment.

A large amount of studies have been carried out to show that EEG signal analysis can be used to monitor human factors e.g., sleepiness, fatigue, inattention, and mental load, etc. [7-11] in driving 
situations. However, while collecting and analyzing the in-vehicle data there are several challenges for instance, light variations, motions, vibrations, individual variation, and time period for monitoring etc. Additionally, EEG signals are often contaminated with artifacts that could mislead the diagnosis result. Therefore, it is important to remove artifacts from the EEG signals. Here EEG artifacts refer to the physiological signals other than the brain activity, such as muscle and ocular signals that contaminate the EEG signals [12,13]. The mental state can be classified using the alpha, beta, theta, and delta rhythm of the EEG power spectrum. During stress situations i.e., when mental concentration and focus become difficult beta waves are highly visible and in relaxed situations alpha waves activates. Theta is visible in drowsiness and delta waves are seen in deep sleep. Wavelet is one of the most popular methods, which has been used in various studies for EEG signal analysis [14-20]. Chaotic dynamics of EEG signals have been analyzed to assess mental load in the paper [21]. Largest Lyapunov exponent (LLE), fractal dimension, and attractor pot are used to examine the chaotic characteristics of the EEG signal during mental load condition. In the aforementioned study, the authors get positive value of LLE for all task conditions. However, they failed to evaluate mental load from the LLE values. Again, different mental states have been classified using the LLE in[22]. Four different mental states namely normal, people subjected to music of his choice (two types of music), and under reflexological stimulation are considered in that study. The results shown that the measures are significantly lower in reflexological stimulation than in the other conditions. In several studies, for example, Elif and her colleagues [23-26] have extracted features using LLE for EEG signal classification.

In this paper, we focus mainly on two of the challenges that are: a) individual---focused decision support and b) short-term (1 minute interval) monitoring of in-vehicle driver stress based on EEG signals. The study includes EEG data collection in lab settings and in real road driving. Other physiological sensor signals e.g., Electrocardiography (ECG), Finger temperature, Skin conductance, and Respiration are also collected as reference. An expert who has more than 20 years of experience as a researcher and clinician in the stress domain classified these reference signals as 'stress' or 'healthy' for each test subject. The proposed system works in 3 phases:

1) Artifact handling: in order to handle EEG artifacts, Independent Component Analysis (ICA) $[27,28]$ has been applied in the first step. Later, double-density wavelet denoising [29-31] has been applied to the independent components (ICs) of the ICA.

2) Feature extraction: modified co-variance, largest Lyapunov exponent (LLE), and wavelet transform have been applied to extract features in both time and frequency domain from the EEG signals.

3) Classification: two Case-Based Reasoning (CBR) classification schemes has been proposed in this study. Here, extracted features from phase 2 are used inone classification scheme and the other is applying fuzzification based case representation and classification. The twoclassification schemes and the fuzzification support to classify each minute LLE value, allowing short interval unsupervised (without any human expert's classification) data analysis and classification.

The rest of the paper is organized as follows: Section 2 presents related work. Section 3, presents concept of the proposed system. Section 4, describes the study design. Section 5 presents the materials and methods where signal preprocessing, feature extraction and case representation for CBR are discussed. Section 6 , discusses the results and experimental work.

Finally, Section 7 ends with a discussion.

\section{RELATED WORK}

A large variety of methods such as statistical, machine learning have been used for clustering and classification of EEG data in different types of projects for stress and mental load. Saidatul et al. [32] have presented the anatomy of stress, correlation between stress and EEG signals, and EEG signal processing including feature extraction and classification. It has also found in the experiment that alpha activity reduces while the level of stress increases. Different mental tasks have been classified using ANN in [33]. Here, EEG signals are recorded while performing three mental tasks i.e., imagination of left hand movement, right hand movement and word generation. EEG signal classification approach has been proposed in[34] using Dempster-Shafer (D-S) theory and K-Nearest Neighbor (KNN) classifier. In the study, to obtain EEG recordings, five different metal tasks have been performed.Here, the authors have compared three different $\mathrm{KNN}$ classifiers namely, voting $\mathrm{KNN}$, distance weighted $\mathrm{KNN}$, and KNN classifier based on $\mathrm{D}-\mathrm{S}$ evidence theory. The results have shown that the D-S theory based KNN classifier has achieved higher classification accuracy than the other two. In our recent work in Begum S. et al. [35] a CRB system to classify EEG signals using Multivariate Multiscale Entropy Analysis (MMSE) algorithm and EEG band power has been presented. The results shown that MMSE-CBR approach could correctly classified stressed and healthy cases up to $84 \%$.

Artificial intelligence and Statistical methods have also been applied in a simulator---based study in 2005, [36] here the drivers have exposed to a drifting--task in a simulator. Here, the performances have measured by a moving-average of the lane position deviation. Another article [37] has also proposed a driver's fatigue recognition model based on D-S 
evidence theory. The authors have reported that most of the driver's fatigue recognition methods are based either on features obtained from visual observation such as eye movement, head movement, and facial expression or non-visual aspects of features such as heart rate, ECG and EEG. The approach of considering one aspect of features is not completely reliable and therefore, the authors have suggested that fatigue recognition could be more accurate when it combines multiple sources of information about driver context, situation, goals and preferences. Systems using other physiological signal based approaches have been proposed in various studies. Reisman [38] has discussed about methods for measuring physiological stress using HRV, blood volume plus, and finger temperature. In the paper [39] a fuzzy c-means algorithm has been proposed using ECG, EMG, skin conductance and respiration signal. Shahina et al., have proposed CBR classification approach for stress diagnosis using HRV and finger temperature [40-42]. Table 1 presents studies using EEG signal for driver monitoring. N/A indicates missing values in the table.

Table 1: Mental state monitoring in driving situation using different algorithms based on EEG signal

\begin{tabular}{|c|c|c|c|c|}
\hline No & Authors & Mental state & Real/Simulator & Methods \\
\hline 1 & M. Haak et al., [43] & Stress & Game simulator & Correlation analysis \\
\hline 2 & $\begin{array}{l}\text { M. A. Manjusha et al., } \\
{[44]}\end{array}$ & Stress & Real road & Artificial Neural Network \\
\hline 3 & Sulaiman N. et al., [45] & Stress & Lab & Statistical analysis \\
\hline 4 & $\begin{array}{l}\text { Schmidt Eike A. et al., } \\
\text { [46] }\end{array}$ & $\begin{array}{l}\text { Alertness, } \\
\text { Driver } \\
\text { Vigilance }\end{array}$ & Real road & $\begin{array}{l}\text { Subjective measures, MANOVA, } \\
\text { Statistical Analysis }\end{array}$ \\
\hline 5 & $\begin{array}{l}\text { Sibsambhu Kar et al., } \\
\text { [47] }\end{array}$ & Fatigue & Real road & Entropy, Energy-based analysis \\
\hline 6 & Schier Mark A., [48] & & Simulator & MANOVA statistical analysis \\
\hline 7 & Lei Cao et al., [49] & Fatigue & Lab & $\begin{array}{l}\text { Principal component analysis, Fisher } \\
\text { score }\end{array}$ \\
\hline 8 & Saroj K. L et al., [50] & Fatigue & Simulator & Statistical analysis \\
\hline 9 & Borghini, G. et al., [51] & Fatigue & Simulator & Statistical analysis \\
\hline 10 & $\begin{array}{l}\text { Paul van den Haak et al., } \\
\text { [52] }\end{array}$ & Stress & Simulator & Statistical analysis \\
\hline 11 & $\begin{array}{l}\text { Venkatesh } \\
\text { Balasubramanian et al., } \\
\text { [53] }\end{array}$ & $\begin{array}{l}\text { Cognitive } \\
\text { fatigue }\end{array}$ & Simulator & $\begin{array}{l}\text { Statistical analysis, Independent } \\
\text { Component analysis, Fast Fourier } \\
\text { transform }\end{array}$ \\
\hline 12 & Saidatul A. et al., [32] & Stress & N/A & Review on several methods \\
\hline 13 & Saidatul A. et al., [54] & Stress & Lab & $\begin{array}{l}\text { Statistical analysis, K-NN, Alpha brain } \\
\text { symmetry score }\end{array}$ \\
\hline 14 & $\begin{array}{l}\text { Shamsul B. M. T. et al., } \\
\text { [6] }\end{array}$ & Stress & Simulator & Statistical analysis \\
\hline 15 & Calibo T. K. et al., [55] & Stress & Lab & K-NN and logistic regression \\
\hline 16 & Merino M. et al., [3] & Stress & Lab & Statistical analysis \\
\hline 17 & Unsoo Ha et al., [56] & Stress & Lab & Support Vector Machine \\
\hline 18 & Bin H. et al., [57] & Stress & Lab & Statistical Analysis \\
\hline
\end{tabular}

However, as the study indicates, EEG signal classification algorithms for in-vehicle stress monitoring is limited. Simulator and lab study is common as a data collection procedure as shown in table 1. Majority of the study use statistical analysis to show a correlation between stress and EEG signal.

The proposed system applies signal processing and artificial intelligence algorithms in real road EEG driving data to monitor in---vehicle stress. Further, it will provide individual---focused solutions in a short time interval. We previously have developed stress diagnosis systems using other physiological sensor signals e.g., ECG, Respiration, Skin conductance and finger temperature $[41,42]$, however, since EEG is one of the most reliable sensor for measuring several other human factors e.g., sleepiness, fatigue, cognitive load it is interesting to investigate the usefulness of EEG signal in this domain $[44,45,15,58]$.

This can be useful to develop a system that fuses several sensors information to provide more robust solution. To our knowledge, system with such functionalities using EEG is limited and this will help 
to advance the development of monitoring systems using EEG signal.

\section{OVERVIEW OF IN-VEHICLE STRESS MONITORING}

An overview of the proposed driver monitoring system based on the EEG signal is presented in Figure 1. Several major steps considered here are: data acquisition, artifact handling, feature extraction and decision support.

\section{Data Acquisition}

Since psychological and behavioural conditions/status that reflect in physiological sensor signals are so personalized individual profiling is therefore an important issue here. Therefore, the first stage of the data collection is to define a standard protocol in the lab settings to establish an individual profile. We followed a 15 minutes protocol that is used for clinical stress measurement. The protocol is described in details in [40]. After profiling, for each individual, data have been collected in a real-driving situation. Along with sensor data contextual information e.g., age, sex, health condition, medication etc. about test subjects have also been collected during this phase. Before the study all the participants sign a letter of consent to agree with the test conditions. The reference physiological parameters e.g., ECG, Respiration, FT and Skin Conductance etc. are collected and considered for the system's evaluation.

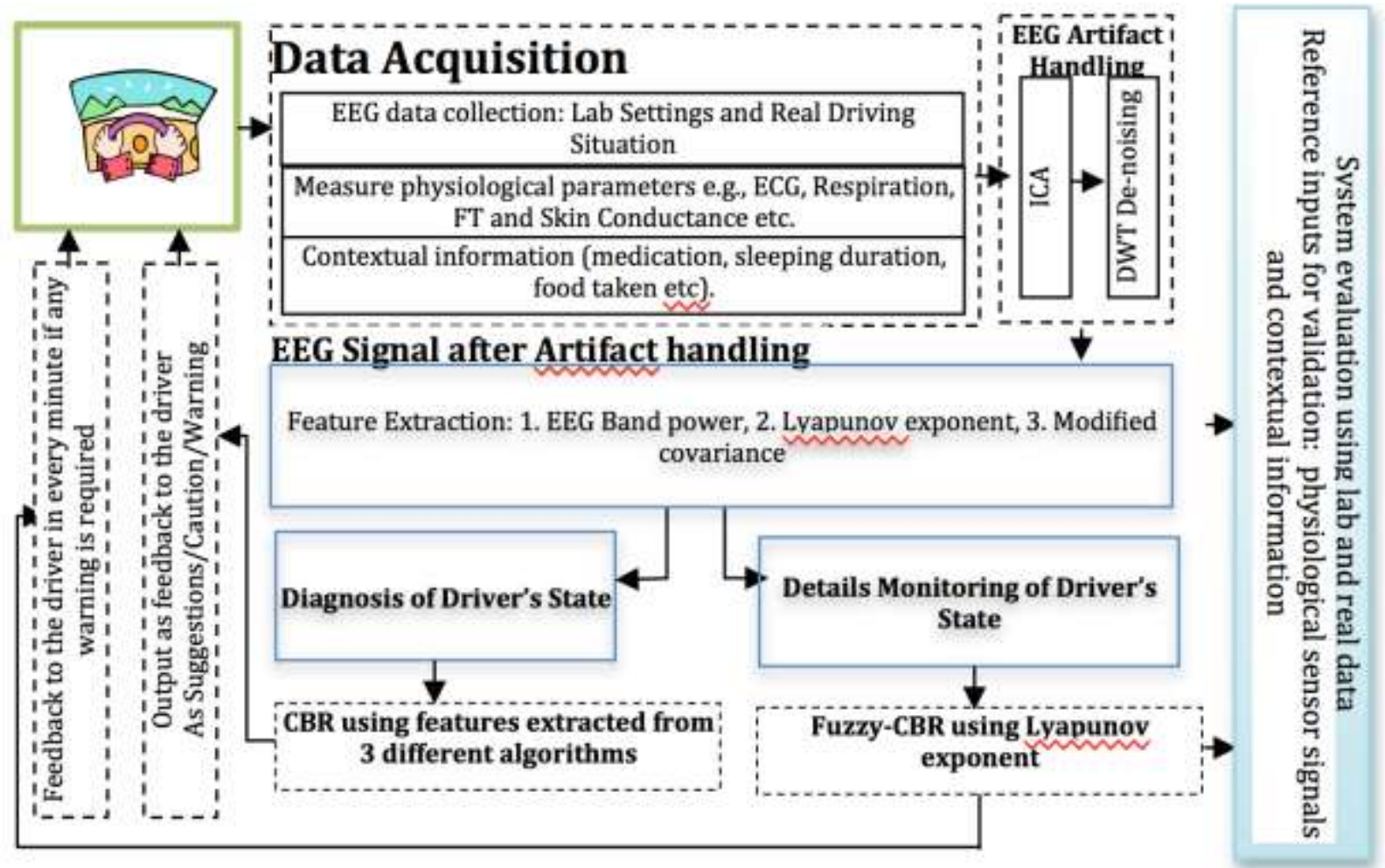

Figure 1: An overview of the proposed driver monitoring system based on EEG signal

\section{Artifact Handling}

EEG is one of the complex sensor measurements and could easily be contaminated with unwanted data for instance, interference from electronic equipment, movements, light, muscle and ocular artifacts etc. This is even more obvious in movement environment such as in driving situation. But, data without handling these artifacts could mislead the analysis result. Algorithms i.e., ICA and Wavelet Denoising are used here for automatic handling of artifacts from the EEG sensor signals.

\section{Feature Extraction}

After artifact handling, the next step is to extract important features fromthe EEG signal that represents the signal. It is usually a promising task to find out key features from a physiological sensor signal. However, due to underlying complexity of the signal feature extraction from EEG signal is difficult. So, a number of approaches namely, discrete wavelet transform, Largest Lyapunov Exponent (LLE) and modified covariance have been investigated to extract quantitative features from the collected EEG signal. Finally, 14 features are extracted to formulate cases in the proposed CBR system.

Lyapunov exponent is a measure that is used to quantify the rate of separation between two neighboring trajectories. In another words, it defines the average rate of divergence or convergence of two neighboring trajectories. It is useful for distinguishing numerous orbits based upon their orientations in the initial conditions. It has been applied to determine the 
chaotic characteristics of the dynamical systems. A system with chaotic characteristics shows aperiodic dynamics because the phase space trajectories, that is closely identical in the initial states become separate from each other at an exponential rate by the Lyapunov exponent. Positive Lyapunov exponent denotes the chaos in a system and shows that neighboring points with infinitesimal differences at the initial state shortly become separated from each other in the i-th direction. The modified covariance is a parametric method and it works on minimizing the forward and backward prediction errors. Modified covariance gives high resolution for short data records and it has ability to extract frequencies from more pure sinusoids. It also produces sharpest peaks. From data it can identify sinusoid components. In the paper $[59,60]$ features are extracted from EEG signals using modified covariance method and suggested that the features obtained from the modified covariance method is better than using eigenvector method such as Pisarenko and Multiple Signal Classification methods.

\section{Decision Support}

Here, decision has been presented in two different ways: one considering the long time duration [5 mins] i.e., overall status of the subject and another is short time interval $\left[\begin{array}{ll}1 & \mathrm{~min}\end{array}\right]$ i.e., detail monitoring information. The advantage of the CBR system is that the system can learn according to user's profile and provide a individual focused solution. So, here the after feature extraction, profile data is taken as input to the CBR system. It provides outcome that is more transparent enabling explanation of the solution and weight values for the features presents the importance of each feature. The system provides decision in a list of possible solutions according to their similarity value. The system can alert in severe situations, if necessary.
In this paper, CBR [61-65] and Fuzzification $[40,66,67]$ has been applied to facilitate decision-making. Retrieval of similar past cases to solve the current problem is the first step of the system. The cases are represented using features extracted from the 3 different methods and fuzzification of six LLE features. The evaluation of the system has been done in comparison with a human expert who has more than 20 years of experience in stress domain as a physician and researcher. The signals are classified as Stressed or Relaxed considering the full-length signal recording based on the other reference parameters i.e., ECG, respiration, heart rate variability, finger temperature, skin conductance etc.

In CBR, a case library is created based on expert knowledge. So, it can learn based on expert's knowledge using EEG features from three different algorithms. Another, artificial case library is developed learning from expert's knowledge. Since, it is not possible for an expert to classify long time series data in real time situation so, the aim here is to learn from the expert's knowledge to provide decision as close as a human expert. Therefore, a fuzzification approach has been introduced to develop an artificial case library considering expert's knowledge. Here, each minute EEG features are extracted using LLE. So, six features from six minutes data of the two data sets i.e., Relaxed and Stressed are fuzzified using the triangular membership function. Later, for Relaxed and Stressed cases, the degree of membership of each LLE values of the real road driving dataset are also calculated using the triangular function.

\section{STUDY DESIGN}

For this study, the data has been collected in two different scenarios:

i) lab settings and ii) real road driving (Figure 2).

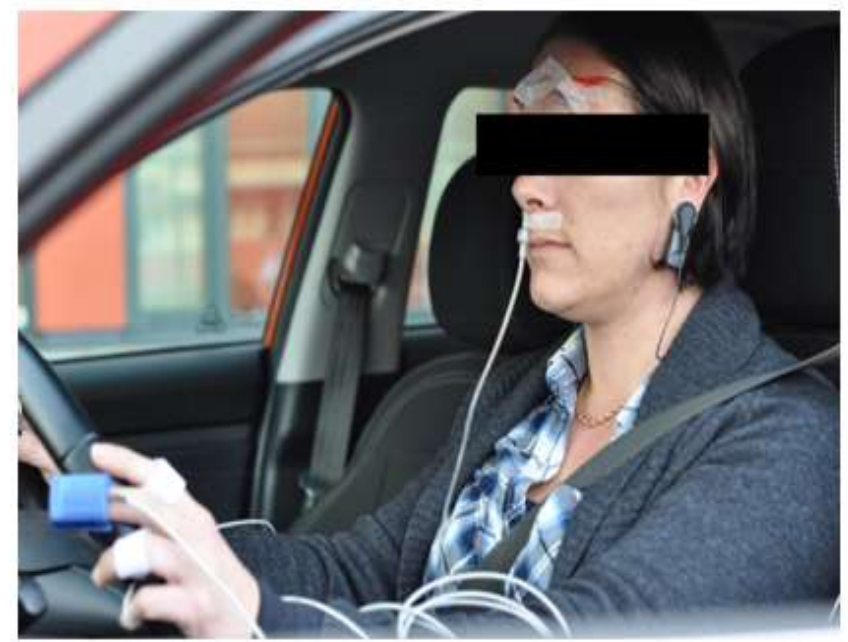

Figure 2: Data collection in real road 
In this study, the data were collected from eight individuals (healthy and medication free) aged between 26-50. Among the participants, seven were male and one was female subject. Here, four of the participants had more than 10 years ofdriving experienced, three of them had approximately five years of driving experience and three were novice drivers. All the participants were informed about the experimental setup before the data collection and contextual data such as age, sleeping duration at night before the data collection, medication driving experience etc., had been collected. These contextual information were used by the expert to classify the individuals. Here, Airpass and C2 devices were used along with the cStress $^{\boldsymbol{l}}$ software to collect the data. Five sensor signals i.e. heart rate (HR), finger temperature $(\mathrm{FT})$, respiration rate $(\mathrm{RR}), \mathrm{CO} 2$, and oxygen saturation (SPO2) were recorded, where Airpass device was used for RR, CO2, and SPO2 signals and C2 device was used for HR, and FT. EEG signal were collected using the NeXus-10 Mark II, the device communicates wirelessly in real--- time. In the EEG data collection the 10-20 systems was used for electrode placement [68]. The electrodes were placed at the locations Fp1, Fp2, Cz (ground), A1 and A2 (references). The EEG data were recorded at a sample rate of $256 \mathrm{~Hz}$. Then the data were filtered using a 1-45 band pass filter. The data collection was carried out in two steps. The first step is, psychophysiological stress profile (PSP) the second step is a real road driving. In the real road driving, the selected route was in the central area of the city with a busy traffic and the distance was approximately 3.5 $\mathrm{km}$. The drivers drove from a start point to a specific end point than 30 seconds break, and drove back again to the start point. Therefore, we obtained two driving sessions data for each of the driver. The data were collected between 3:30 PM and 4:30 PM in the evening during the office hour. This time was selected because of assessing stress level in heavy road traffic. A five minute time constrain was imposed for each driving session and after the four minutes in every ten seconds interval drivers' were informed about the time left to reach the destination.

\section{METHODS AND APPROACHES}

In this paper, the recorded EEG signals are pre-processed and then artifacts are handled in two steps: first, the Independent Component Analysis (ICA) [27,28] has been applied and later double-density wavelet denoising [29-31] is performed on the independent components that are obtained from ICA. ICA is the most common method that has been applied for artifacts handling in EEG signal. However, one of the drawbacks with ICA is that it needs visual inspection to extract artificial components. Therefore, Wavelet denoising has been combined with ICA to suppress artifacts from the EEG signals [69-72].

\section{Feature Extraction and Case Representation}

Feature extraction is one of the major tasks in this project. Several algorithms have been applied to extract important features from the collected EEG signals. Here, discrete wavelet transform (DWT) is used to calculate the Four frequency bands, Largest Lyapunov exponent (LLE) calculates each minute EEG signal and give the exponential value as features and Modified co-variance is applied to calculate the statistical features such as, maximum, minimum, standard deviation, and average.

\section{Discrete wavelet transform (DWT)}

Physiological signal such as EEG is time varying and has complex time---frequency characteristics. Time-varying physiological signals are often analysed using the wavelet transform, which expends the signals into basis functions. A wavelet basis function $a, b(t)$ is generated by dilation, translation and shift of a unique function called mother wavelet $(t)$.

$$
{ }_{a, t}(t)=\frac{1}{\sqrt{|a|}}\left(\frac{t b}{a}\right)
$$

Where, $a, b$ are the scaling dilation and transition parameters and $t$ represents time. Wavelet length and oscillatory frequency are determined by dilation parameter, and shifting position decides by the translation parameter. EEG waveforms can be classified into several rhythmic frequencies that are influenced by the mental states. The four frequency bands that are frequently used for stress and mental load classification are alpha $(8-12 \mathrm{~Hz})$, beta $(13-30$ $\mathrm{Hz})$, theta $(4-8 \mathrm{~Hz})$, and delta $(0-4 \mathrm{~Hz})$. Here, the

EEG signals are decomposed using the Daubechies wavelet family function ('db4') and then the power spectral density (PSD) is calculated for each of these four---frequency bands. Since sample rate of the EEG signals is $256 \mathrm{~Hz}$, level-6 decomposition is applied here.

Largest Lyapunov exponent (LLE) EEG signal recorded from one site of the brain is associated with the co-called embedding phase space. EEG data $x(t)$ of duration $\mathrm{T}$ can be defined by the vector $x_{i}$ in the phase space as shown in Eq. (2)

$$
x_{i}=\left\lfloor x\left(t_{i}\right), x\left(t_{i}+\right), \ldots \ldots, x\left(t_{i}+\left(\begin{array}{ll}
p & 1
\end{array}\right)\right)\right\rfloor
$$


Where, $\mathrm{p}$ is the selected dimension of the embedding space, represents selected time lag between the components of each vector and $t_{i}$ is the

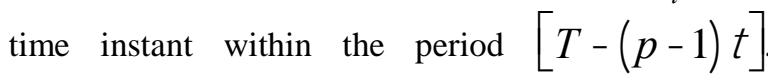
Consider two EEG data points $X_{0} X$ and $X_{0}+x_{0} X$ in a space. Also consider that these two points will generate an orbit in that space and the separation between them is $x$. This separation has the form $x\left(X_{0}, t\right)$ and will behave erratically.
The mean exponential rate of divergence of the two initially close orbits is given in Eq. (3)

$$
L E=\lim _{t \rightarrow \infty} \frac{1}{t} \ln \frac{\left|x\left(X_{0}, t\right)\right|}{\left|X_{0}\right|}
$$

Where, $L E$ is the Lyapunov exponent and the maximum value of $L E$ is called LLE. Lyapunov exponent measures the rate of the divergence of nearby trajectories in a dynamical system. In this study Rosenstein's algorithm [73] is used to extract LLE feature from the EEG signal.

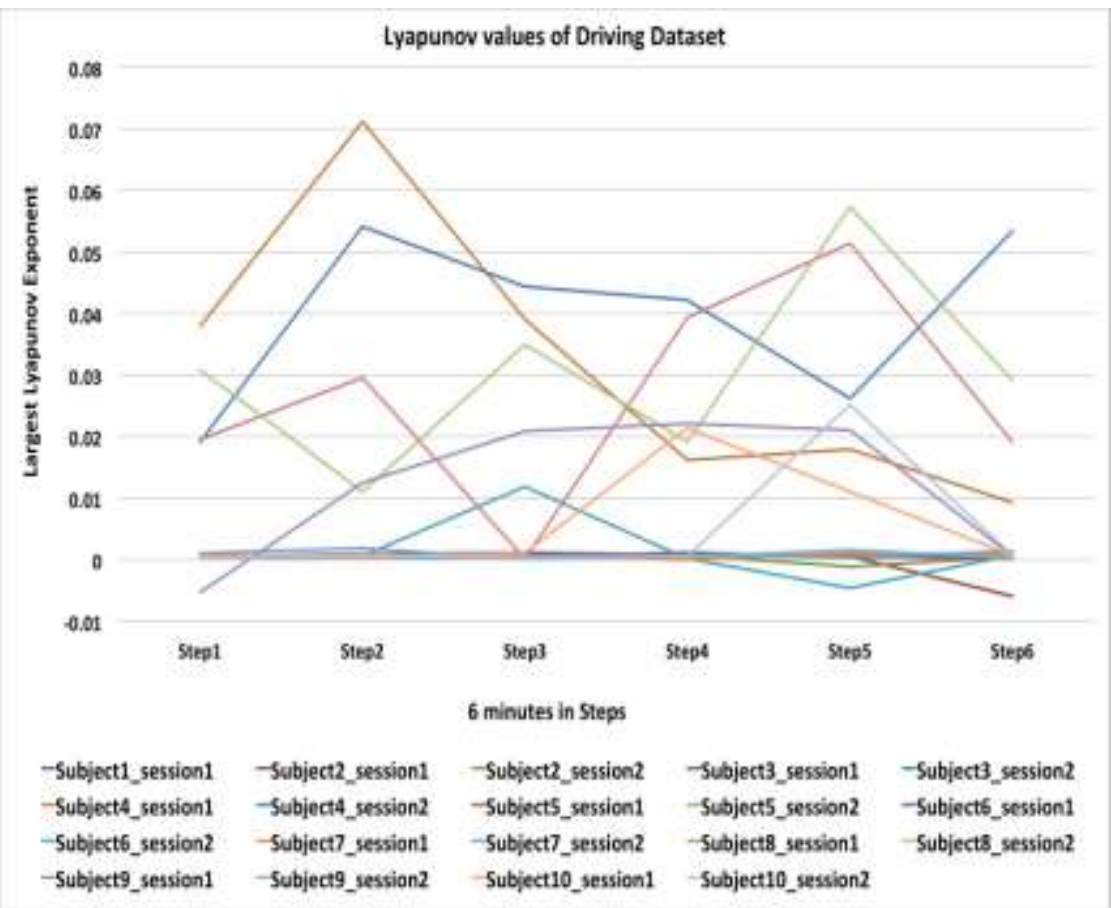

Figure 3: Lyapunov features variation in driving session data

Figure 3 presents Lyapunov exponent for 10 individuals during the 2 driving sessions. X-axis of the graph represents the 6 minutes driving divided in to 6 steps (each step is one minute) and Y-axis represents the corresponding Lyapunov exponent estimated from each minute EEG data. In Figure 3, most of the data show chaotic characteristics and it is difficult to make any general rule from these signals to classify drivers' stress.

\section{Modified Covariance}

In the parametric methods, one approach of estimating autoregressive model parameters is the modified covariance method. In the modified covariance method autoregressive parameters are found by solving the Eq. (4) and Eq. (5).

where,

$$
\left[\begin{array}{cccc}
r_{x}(1,1) & r_{x}(2,1) & \cdots & r_{x}(p, 1) \\
r_{x}(1,2) & r_{x}(2,2) & \cdots & r_{x}(p, 2) \\
\vdots & \vdots & \vdots & \vdots \\
r_{x}(1, p) & r_{x}(2, p) & \cdots & r_{x}(p, p)
\end{array}\right]\left[\begin{array}{c}
a_{p}(1) \\
a_{p}(2) \\
\vdots \\
a_{p}(p)
\end{array}\right]=-\left[\begin{array}{c}
r_{x}(0,1) \\
r_{x}(0,2) \\
\vdots \\
r_{x}(0, p)
\end{array}\right]
$$

$$
r_{x}(k, l)=\sum_{n=p}^{N-1}\left[x(n-l) x^{*}(n-k)+x(n-p+l) x^{*}(n-p+k)\right]
$$


Here $r_{x}(k, l)$ is the autocorrelation, $a_{p}$ is the autoregressive parameter, $x$ is the complex conjugate of $x, p$ is model order, $N$ number of data points.

In this study, MatLab function pmcov has been applied to the EEG signal to obtainpower spectral density (PSD) using modified covariance method. Then, the statistical features i.e., maximum, minimum, mean, and standard deviation of PSDs are calculated as features.
Table 2 shows the list of features using the three different algorithms i.e., band power, modified covariance and Lysponuv exponent. The weight values for the band power are determined based on the literature review $[26,74,45]$. Here, the weights for the Lysponuv exponents for the collected six minutes signal are selected considering the each minute data analysis. For the modified covariance, weights values are given same for all the features.

Table 2: List of features using band power, modified covariance, and Lyapunov exponent

\begin{tabular}{|c|c|c|}
\hline & Feature values & Weight \\
\hline \multirow{5}{*}{ Band Power } & Alpha & 8 \\
\hline & Beta & 10 \\
\hline & Theta & 6 \\
\hline & Delta & 1 \\
\hline & Maximum & 1 \\
\hline \multirow{3}{*}{$\begin{array}{l}\text { Modified } \\
\text { Covariance }\end{array}$} & Minimum & 1 \\
\hline & Mean & 1 \\
\hline & Standard deviation & 1 \\
\hline \multirow{6}{*}{ Lyapunov } & $\begin{array}{c}\text { Exponential value of } 1 \\
\text { minute }\end{array}$ & $\overline{1}$ \\
\hline & $\begin{array}{c}\text { Exponential value of } \\
2^{\text {nd }} \text { minute }\end{array}$ & 10 \\
\hline & $\begin{array}{c}\text { Exponential value of } \\
3^{\text {rd }} \text { minute }\end{array}$ & 8 \\
\hline & $\begin{array}{c}\text { Exponential value of } \\
4^{\text {th }} \text { minute }\end{array}$ & 1 \\
\hline & $\begin{array}{c}\text { Exponential value of } \\
5^{\text {th }} \text { minute }\end{array}$ & 5 \\
\hline & $\begin{array}{c}\text { Exponential value of } \\
6^{\text {th }} \text { minute }\end{array}$ & 10 \\
\hline
\end{tabular}

\section{The Proposed Classification Approach}

In this study, CBR classification has been done using the extracted features and case formulation has been performed based on fuzzification. Figure 4 illustrates the CBR scheme using the extracted features and Figure 5 describes the Fuzzy-CBR classification scheme. It shows in Figure 4 that after the data collection, raw EEG signals are pre-processed and artifacts handling has been performed. Then the features are extracted using the DWT, modified covariance, and LLE (see section 5.2) and these extracted features are used to build the three separate case libraries for the CBR classification. Finally, the combined result is obtained considering the weighted majority-voting algorithm. Here, these weight values are defined based on the individual classification result. 


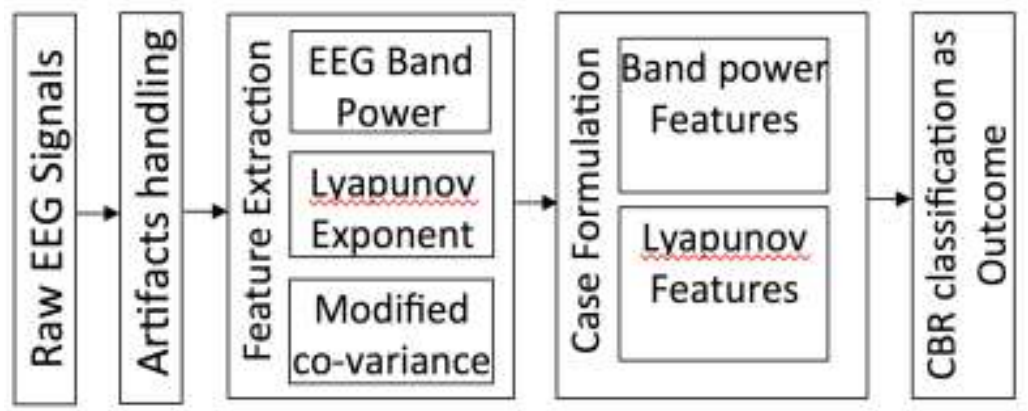

Figure 4: An overview of the CBR classification scheme using the extracted features

On the other hand, in the fuzzy---CBR classification scheme as depicted in Figure 5, fuzzification has been done on LLE values for Relaxed and Stressed cases using the Fuzzy Triangular membership function. Then using the fuzzified values a case library has been developed for the CBR classification.

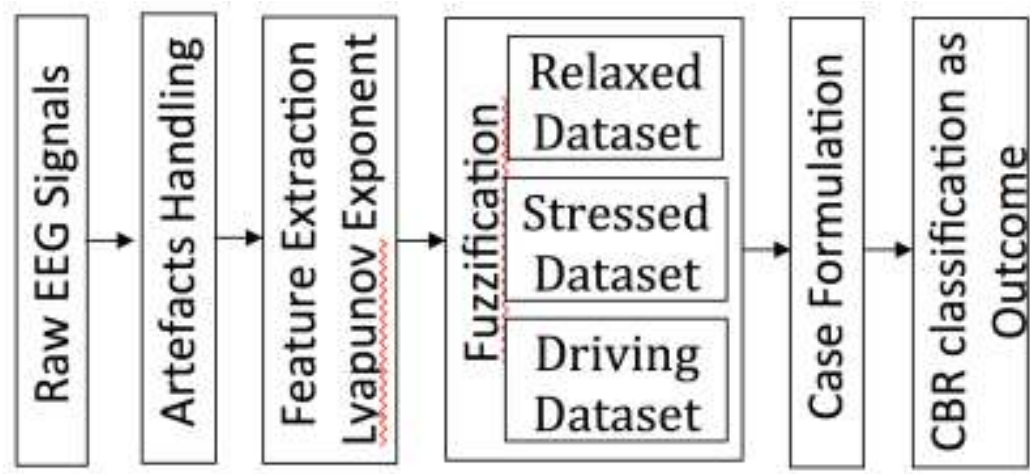

Figure 5: An overview of the Fuzzy-CBR classification Approach

Figure 6 presents average of the Lyapunov exponent for the Relaxed, Stressed classes, and Driving data. It can be seen from Figure 2 that the data sets are distinguishable. However, when we consider all the data from each individual then there is an overlap between these feature values and it is not possible to make any general rule. Therefore, the idea is to apply fuzzification to classify each feature values as Relaxed and Stressed. Fuzzification transforms crisp quantity into grades of membership of fuzzy sets. Here, the crisp Stressed and Relaxed values are fuzzified. The Triangular membership function has been applied on the Lyapunov features of Relaxed and Stressed datasets.

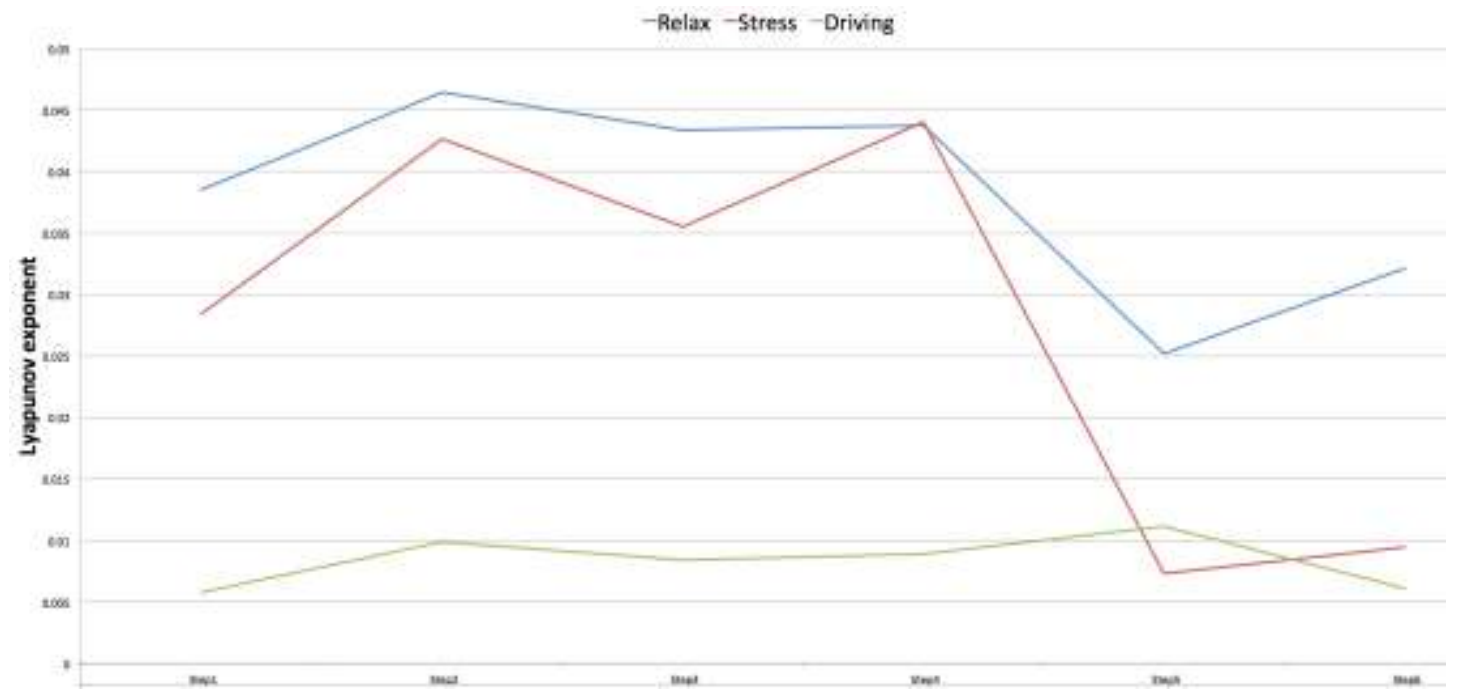

Figure 6: Average of Lyapunov exponent for relaxed, stressed, and driving situation datasets 
Then the membership degree for each LLE value of Relaxed and Stressed cases are obtained from this. Thus, for these triangular membership functions two vectors i.e., Relaxed and Stressed are obtained. Thereafter, maximum values for each element of these two vectors are considered and cases are classified either as Stressed or Relaxed depending on the degree of the membership function. Then, the Driving dataset is also classified using the fuzzy models and the results are presented in the later section. In this study, for both the stressed and relaxed group Lyapunov feature values are sorted and then the statistical measures i.e., maximum, minimum and average values of LLE values are estimated to generate the fuzzy triangular membership function (MF); so that it satisfies the core or center of the MF i.e., $x \quad{ }_{i}(x)=1$, and the footprint or the support of the MF $x \quad(x)>0[75]$

\section{EXPERIMENTAL WORK}

Several experimental works have been carried out to evaluate the proposed approach. In the first step, three separate case libraries have been created using the features obtained from EEG band power, Modified covariance and Lyapunov exponent. These case libraries contains 57 cases consisted of relaxed, stressed, and driving dataset. Here, it is important to mention that the expert has classified each case of the driving dataset as either Stressed or Relaxed. Later, the
Lyapunov exponent features have been fuzzified for Relaxed and Stressed classes. Then, feature values from both of these datasets are used to obtain the degree of membership function. The membership functions are generated by trail and error. Here, the cases are not classified by any human expert rather it has been done automatically using fuzzification. However, the accuracy of the case-library has been evaluated in a CBR system and presented the result by comparing with the expert.

For the case retrieval, a "leave-one-out" retrieval technique has been applied i.e., one case is taken out from the case library as a query case. Here, $\mathrm{kNN}(\mathrm{K}=2)$ is applied to retrieve similar cases. For the evaluation, two top most similar retrieved cases have been considered; if both the query and one of the two retrieved cases belonging to a similar class then the number of correctly classified cases is counted as 1 .

\section{CBR Classification using extracted features}

The summary of the CBR classification based on EEG band power, modified covariance and Lyapunov exponent features are presented in Table 3. It can be seen from Table 3, for band power features, considering the top most one case, i.e., $\mathrm{k}=1 \mathrm{CBR}$ accuracy for Relaxed and Stressed cases are $46.15 \%$ and $48.29 \%$. While, considering $\mathrm{k}=2$ the achieved accuracies are $61.54 \%$ for Relaxed and $83.87 \%$ for Stressed cases.

Table 3: CBR classification using EEG band power, modified covariance, and Lyapunov features

\begin{tabular}{lllllll}
\hline \hline Case Classes & \multicolumn{2}{l}{ Band power } & \multicolumn{2}{l}{ Modified Covariance } & \multicolumn{2}{l}{ Lyapunov exponent } \\
\hline Criteria/Indices & $\mathrm{K}=1$ & $\mathrm{~K}=2$ & $\mathrm{~K}=1$ & $\mathrm{~K}=2$ & $\mathrm{~K}=1$ & $\mathrm{~K}=2$ \\
26 Relaxed & $46.15 \%$ & $61.54 \%$ & $65.38 \%$ & $88.46 \%$ & $46.15 \%$ & $73.07 \%$ \\
31 Stressed & $48.29 \%$ & $83.87 \%$ & $54.84 \%$ & $87.09 \%$ & $61.29 \%$ & $90.32 \%$ \\
Total 57 cases & $47.37 \%$ & $73.68 \%$ & $70.18 \%$ & $87.72 \%$ & $54.39 \%$ & $82.46 \%$ \\
\hline \hline
\end{tabular}

On the other hand, using the modified covariance features, when $\mathrm{K}=1$, the achieved accuracy is $65.38 \%$ for Relaxed and $54.84 \%$ for Stressed cases. While for $\mathrm{K}=2$, it is $88.46 \%$ for Relaxed and $87.09 \%$ for Stressed cases. Furthermore, using Lyapunov features, when $\mathrm{k}=1$, the obtained accuracy is $46.15 \%$ for Relaxed and $61.29 \%$ for Stressed classes. Considering $\mathrm{K}=2$, the accuracies are obtained as $73.07 \%$ and $90.32 \%$ forRelaxed and Stressed cases respectively.

Table 4 demonstrates CBR classification comparison among EEG band power, modified covariance and Lyapunov features. Here, sensitivity, specificity and overall accuracy are also calculated. It can be seen from Table 3 using the band power features, out of 31Stressed cases 26 are correctly classified and 16 Relaxed cases are correctly classified from the 26 cases. In contrast, Modified co-variance has classified 27 Stressed and 23 Relaxed cases correctly. On the other hand, using the Lyapunov features 28 Stressed and 19 Relaxed cases are correctly classified. While, 25 Stressed and 22 Relaxed cases are correctly applied the voting algorithm. In this table, sensitivity i.e., percentage of cases that are identified as Stressed, is $84 \%$ for band power, $87 \%$ for modified covariance and $90 \%$ for Lyapunov features. Specificity i.e., percentage of cases that are identified as Relaxed is $62 \%$ for band power, $88 \%$ for modified covariance and $73 \%$ for Lyapunov features; and the overall accuracies are $74 \%$ for band power, $88 \%$ for modified covariance and $82 \%$ for Lyapunov features. After voting, for the proposed system, the combined result gives an accuracy of $82 \%$. 
Table 4: Sensitivity and Specificity analysis comparing the band power, modified covariance, and Lyapunov exponent features. The last column presents the voting result considering the three features when $\mathrm{K}=2$

\begin{tabular}{lllll}
\hline \hline & BAND PowER & Modified Covariance & Lyapunov exponent & Voting \\
\hline \hline Criteria/Indices & $\mathrm{K}=2$ & $\mathrm{~K}=2$ & $\mathrm{~K}=2$ & $\mathrm{~K}=2$ \\
\hline Total cases & 57 & 57 & 57 & 57 \\
Cases belong to Stressed group (P) & 31 & 31 & 31 & 31 \\
Cases belong to Relaxed group (N) & 26 & 26 & 26 & 26 \\
True positive (TP): & 26 & 27 & 28 & 25 \\
False positive (FP): & 10 & 3 & 7 & 4 \\
True negative (TN): & 16 & 23 & 19 & 22 \\
False negative (FN): & 5 & 4 & 3 & 6 \\
Sensitivity = TP / (TP + FN) & $\approx 0.84$ & $\approx 0.87$ & $\approx 0.90$ & $\approx 0.81$ \\
Specificity = TN / (FP + TN) & $\approx 0.62$ & $\approx 0.88$ & $\approx 0.73$ & $\approx 0.85$ \\
Accuracy = (TP+TN)/(P+N) & $\approx 0.74$ & $\approx 0.88$ & $\approx 0.82$ & $\approx 0.82$ \\
\hline \hline
\end{tabular}

\section{Fuzzy-CBR classification}

\section{Using Profile Data}

For the fuzzy case representation using Fuzzy Triangular function Table 5 shows the CBR classification accuracy. It can be seen from Table 5 that 94 feature values are classified as relaxed and 134 feature values are classified as Stressed using the Triangular fuzzy function. Considering $\mathrm{k}=2 \mathrm{CBR}$ classification accuracy is obtained as $65 \%$ for Relaxed and $86 \%$ for Stressed cases.

Table 5: Fuzzy Triangular membership function using Lab setting data

\begin{tabular}{|c|c|c|}
\hline \multicolumn{3}{|l|}{ Case Classes } \\
\hline Criteria/Indices & $K=1$ & $K=2$ \\
\hline 94 Relaxed & $43.82 \%$ & $65.17 \%$ \\
\hline 134 Stressed & $61.15 \%$ & $86.33 \%$ \\
\hline Total 228 coses & $54.39 \%$ & $78.07 \%$ \\
\hline
\end{tabular}

\section{Using Driving Data}

Now, when the driving dataset have been applied using only the fuzzification it classifies 26 cases as Relaxed and 88 cases as Stressed. However, to evaluate the accuracy of fuzzy classification and to get advantage of the CBR system the combined approach Fuzzy-CBR has been evaluated. Driving dataset has been classified using the CBR library (discussed in Table 5). When CBR classification is applied using fuzzified case library then out of 114 feature values of driving dataset 68 feature values are classified as Relaxed.

From Figure 6, it is noticeable that average Driving dataset is lower than the Relaxed dataset and the expert also has classified most of the driving cases as Stressed, and after fuzzification the fuzzified caselibrary also reflects this characteristic. This new case library is then evaluated and the evaluation result is shown in Table 6. Here, the sensitivity, specificity and overall accuracy are $92 \%, 57 \%$ and $79 \%$ respectively.

Table 6: Classification of driving data using Fuzzy-CBR approach

\begin{tabular}{lll}
\hline \hline Criteria/Indices & $\mathrm{K}=1$ & $\mathrm{~K}=2$ \\
\hline Total cases & 19 & 19 \\
Cases belong to Stressed group (P) & 12 & 12 \\
Cases belong to Relaxed group (N) & 7 & 7 \\
True positive (TP): & 10 & 11 \\
False positive (FP): & 5 & 3 \\
True negative (TN): & 2 & 4 \\
False negative (FN): & 2 & 1 \\
Sensitivity = TP / (TP + FN) & $\approx 0.83$ & $\approx 0.92$ \\
Specificity = TN / (FP + TN) & $\approx 0.29$ & $\approx 0.57$ \\
Accuracy = (TP+TN)/(P+N) & $\approx 0.63 \approx 0.79$ \\
\hline \hline
\end{tabular}

\section{Comparison with Other Physiological signals}


Table 7 presents comparison of stress classification using EEG and other physiological signals i.e., heart rate variability (HRV), finger temperature (FT), and skin conductance (SC). In time domain, HRV features are extracted from statistical measures of inter-beat-interval (IBI) signal and in frequency domain, power spectral density is estimated applying the First Fourier Transformation [42]. FT and SC features are extracted using a derivative of slop based method, details can be found in [76]. The same dataset with 57 EEG cases have been compared with their corresponding cases formulated from the features obtained using the HRV, FT and SC signals. It can be seen from Table 7 classification accuracy using $\mathrm{HRV}$ is $87.79 \%$, FT is $77.19 \%$ and SC is $75.43 \%$. Only the accuracy with the HRV is close to the accuracy using EEG signal.

Table 7: Classification comparison among different Physiological Signals

\begin{tabular}{lccclll}
\hline \hline No & $\begin{array}{l}\text { HRV } \\
(\%)\end{array}$ & $\begin{array}{l}\text { FT } \\
(\%)\end{array}$ & $\begin{array}{l}\text { SC } \\
(\%)\end{array}$ & $\begin{array}{l}\text { EEG } \\
\text { Bandpower } \\
(\%)\end{array}$ & $\begin{array}{l}\text { EEG } \\
\text { Modified Covariance } \\
(\%)\end{array}$ & $\begin{array}{l}\text { EEG } \\
\text { Lyapunov } \\
(\%)\end{array}$ \\
\hline \hline $\begin{array}{l}\text { 31 Stressed } \\
\text { cases }\end{array}$ & 87.09 & 67.74 & 67.74 & 83.87 & 87.72 & 90.32 \\
$\begin{array}{l}\text { 26 Relaxed } \\
\text { cases }\end{array}$ & 88.46 & 88.46 & 88.46 & 61.54 & 87.69 & 73.07 \\
Accuracy & 87.79 & 77.19 & 75.43 & 73.68 & 87.72 & 82.46 \\
\hline
\end{tabular}

\section{DISCUSSION}

Decreased vehicle control due to different driver's mental state is one of the main reasons of road accident. This paper proposed an approach in identifying individual response during mental stress using EEG signals. In this study, the data collection has been done in two steps; one is the lab setting (profile) and the later is the real road driving. The aim is to collect data during stressed driving situation. Features are extracted from EEG signals using DWT, Modified Co- variance and LLE algorithm, and later the CBR classification has been performed. One of the goals of the investigation is to classify the data without an expert's involvement. Here, a human expert has classified the profile datasets using the reference physiological sensor signals. Then, the CBR system has been developed using the reference caselibrary; where the case-library has been built using the features extracted from the three mentioned algorithms. However, in such systems it is necessary that the classification has been done unsupervised i.e., without expert's involvement. Therefore the crisp values of Lyaponuv exponent have been fuzzified using Triangular membership function. This fuzzification allows handling uncertainties in decisionmaking. Lyaponuv exponent shows distant characteristics for Stressed and Relaxed cases for each minute interval. It also presents in Figure 4 that the signals do not show any general rules to extract.

In our previous work $[77,78]$ comparisons have been performed between CBR and other machine learning algorithms, namely, support vector machines (SVM) and artificial neural networks (ANN); and it is found that CBR classification accuracy was better than the SVM and ANN. Therefore, since CBR works well for weak domain theory it has been chosen for decision- making tasks. Moreover, it helps to provide a personalized solution based on the case-library. New knowledge can be added into the case-library that allows learning facilities into the decision-making tasks. The combined approach Fuzzy-CBR provides flexibility in feature values to use it in the reasoning process that handles uncertainties in decision-making.

The result shows that it provides personalized solution with an accuracy of $80 \%$ compare to the expert considering the proposed short time interval. Moreover, it is possible to provide details status of each subject during the driving situation, that is, for example, what is the mental status in each minute or shorter time duration, say in 30 seconds. The result shows that the combined approach has potential for advancing decision support system in monitoring drivers' mental stress. The result could be further improved by optimizing the 'weight values' of the features used to formulate the cases in the case library. It is also important to make a large-scale study to conclude the result.

\section{ACKNOWLEDGMENTS}

The authors would like to acknowledge the Swedish Knowledge Foundation (KKs) and VINNOVA to support research projects in driver monitoring areas and the participated subjects for their contributions 


\section{REFERENCES}

[1]. Subha, D.P., Joseph, P., Acharya U, R., Lim, C.: EEG Signal Analysis: A Survey. J Med Syst34(2), 195-212 (2010). doi:10.1007/s10916-008-9231-z

[2]. Ali, B., Mehrdad, F., Rabab, K.W., Gary, E.B.: A survey of signal processing algorithms in braincomputer interfaces based on electrical brain signals. Journal of Neural Engineering4(2), R32 (2007).

[3]. Merino, M., Gomez, I., Molina, A.J.: EEG feature variations under stress situations. In: Engineering in Medicine and Biology Society (EMBC), 2015 37th Annual International Conference of the IEEE, 25-29 Aug. 2015 2015, pp. 6700-6703

[4]. Tyson, P.: Task-related stress and EEG alpha biofeedback. Biofeedback and Self-Regulation 12(2), 105- 119 (1987). doi:10.1007/BF01000012

[5]. Norhazman, H., Zaini, N.M., Taib, M.N., Omar, H.A., Jailani, R., Lias, S., Mazalan, L., Sani, M.M.: Behaviour of EEG Alpha Asymmetry when stress is induced and binaural beat is applied. In: Computer Applications and Industrial Electronics (ISCAIE), 2012 IEEE Symposium on, 3-4 Dec. 2012 2012, pp. 297-301

[6]. Shamsul, B.M.T., S. Khairunnisa, Ng, Y.G., Syah, M.Y.I.: Stress; the vulnerability and association with driving performance. American Journal of Applied Sciences 11(3), 448-454 (2014). doi:10.3844/ajassp.2014.448.454

[7]. Åkerstedt, T., Knutsson, A., Westerholm, P., Theorell, T., Alfredsson, L., Kecklund, G.: Mental fatigue, work and sleep. Journal of Psychosomatic Research 57(5), 427-433 (2004).doi:http://dx.doi.org/10.1016/j.jpsycho res.2003.12.001

[8]. Michail, E., Kokonozi, A., Chouvarda, I., Maglaveras, N.: EEG and HRVmarkers of sleepiness and loss of control during car driving. In: Engineering in Medicine and Biology Society, 2008. EMBS 2008. 30th Annual International Conference of the IEEE, 20-25 Aug. 2008 2008, pp. 2566--- 2569

[9]. Sulaiman, N., Taib, M.N., Aris, S.A.,Hamid, N.H.A., Lias, S., Murat, Z.H.: Stress features identification from EEG signals using EEG Asymmetry \& Spectral Centroids techniques. In: Biomedical Engineering and Sciences (IECBES), 2010 IEEE EMBS Conference on, Nov. 30 2010-Dec. 22010 2010, pp. 417- 421

[10]. Zahra, M., Miri, A.S.N., Mohammad, M.: EEG--- based Drowsiness Detection for Safe Driving Using Chaotic Features and Statistical Tests. Journal of Medical Signals and Sensors1(2), 130-137 (2011).

[11]. Åkerstedt, T., Hallvig, D., Anund, A., Fors, C., Schwarz, J., Kecklund, G.: Having to stop driving at night because of dangerous sleepiness - awareness, physiology and behaviour. Journal of Sleep Research 22(4), 380--- 388 (2013). doi:10.1111/jsr.12042

[12]. Migotina, D., Calapez, A., Rosa, A.: AutomaticArtifacts Detection and Classification in Sleep EEG Signals Using Descriptive Statistics and Histogram Analysis: Comparison of Two Detectors. Paper presented at the Spring Congress on Engineering and Technology (S-CET), 27-30 May 2012

[13]. Talsma, D.: Auto-adaptive averaging: Detecting artifacts in event-related potential data using a fully automated procedure. Psychophysiology 45(2), 216-228 (2008). doi:10.1111/j.1469-8986.2007.00612.x

[14]. Gandhi, T., Panigrahi, B.K., Anand, S.: A comparative study of wavelet families for EEG signal classification. Neurocomputing 74(17), 3051-3057 (2011). doi:http://dx.doi.org/10.1016/j.neucom.2011.0 4.029

[15]. Wang, X.-W., Nie, D., Lu, B.-L.: Emotional state classification from EEG data using machine learning approach. Neurocomputing 129(0), 94-106 (2014). doi:http://dx.doi.org/10.1016/j.neucom.2013.0 6.046

[16]. Samar, V.J., Bopardikar, A., Rao, R., Swartz, K.: Wavelet Analysis of Neuroelectric Waveforms: A Conceptual Tutorial. Brain and Language 66(1), 7-60 (1999). doi:http://dx.doi.org/10.1006/brln.1998.2024

[17]. Subasi, A.: Automatic recognition of alertness level from EEG by using neural network and wavelet coefficients. Expert Systems with Applications 28(4), 701-711 (2005). doi:http://dx.doi.org/10.1016/j.eswa.2004.12.0 27

[18]. Subasi, A.: EEG signal classification using wavelet feature extraction and a mixture of expert model. Expert Systems with Applications 32(4), 1084-1093 (2007). doi:http://dx.doi.org/10.1016/j.eswa.2006.02.0 05

[19]. Jahankhani, P., Kodogiannis, V., Revett, K.: EEG Signal Classification Using Wavelet Feature Extraction and Neural Networks. In: Modern Computing, 2006. JVA '06. IEEE John Vincent Atanasoff 2006 International Symposium on, 3-6 Oct. 2006 2006, pp. 120124

[20]. Übeyli, E.D.: Wavelet/mixture of experts network structure for EEG signals classification. Expert Systems with Applications 34(3), 1954-1962 (2008). doi:http://dx.doi.org/10.1016/j.eswa.2007.02.0 06 
[21]. Murata, A., Iwase, H.: Analysis of chaotic dynamics in EEG and its application to assessment of mental workload. In: Engineering in Medicine and Biology Society, 1998. Proceedings of the 20th Annual International Conference of the IEEE, 29 Oct1 Nov 1998 1998, pp. 1579-1582 vol.1573

[22]. Natarajan, K., Acharya U, R., Alias, F., Tiboleng, T., Puthusserypady, S.: Nonlinear analysis of EEG signals at different mental states. BioMedical Engineering OnLine 3(1), 7 (2004).

[23]. Übeyli, E.D.: Lyapunov exponents/ probabilistic neural networks for analysis of EEG signals. Expert Systems with Applications 37(2), 985-992 (2010). doi:http://dx.doi.org/10.1016/j.eswa.2009.05.0 78

[24]. Übeyli, E.D.: Modified mixture of experts employing eigenvector methods and Lyapunov exponents for analysis of electroencephalogram signals. Expert Systems 26(4), 339-354 (2009). doi:10.1111/j.14680394.2009.00490.x

[25]. Güler, N.F., Übeyli, E.D., Güler, İ.: Recurrent neural networks employing Lyapunov exponents for EEG signals classification. Expert Systems with Applications 29(3), 506514

(2005). doi:http://dx.doi.org/10.1016/j.eswa.2005.04.0 11

[26]. Übeyli, E.D.: Statistics over features: EEG signals analysis. Computers in Biology and Medicine39(8), 733-741 (2009). doi:http://dx.doi.org/10.1016/j.compbiomed.2 009.06.001

[27]. Delorme, A., Makeig, S.: EEGLAB: an open source toolbox for analysis of single-trial EEG dynamics including independent component analysis. Journal of Neuroscience Methods 134(1), 9-21 (2004). doi:http://dx.doi.org/10.1016/j.jneumeth.2003. 10.009

[28]. Delorme, A., Sejnowski, T., Makeig, S.: Enhanced detection of artifacts in EEG data using higher-order statistics and independent component analysis. NeuroImage 34(4), 14431449

(2007). doi:http://dx.doi.org/10.1016/j.neuroimage. 20 06.11 .004

[29]. Selesnick, I.W.: The double-density dual-tree DWT. Signal Processing, IEEE Transactions on 52(5), 1304-1314 (2004). doi:10.1109/TSP.2004.826174

[30]. Selesnick, I.W.: The Double Density DWT. In: Petrosian, A., Meyer, F.G. (eds.) Wavelets in Signal and Image Analysis: From Theory to Practice. MA: Kluwer, Boston (2001)
[31]. Abdullah, H., Cvetkovic, D.: Double Density Wavelet for EEG Signal Denoising. Paperpresented at the 2nd International Conference on Machine Learning and Computer Science (IMLCS'2013) August 2526, 2013

[32]. Saidatul, A., Paulraj, M.P., Yaacob, S., Mohamad Nasir, N.F.: Automated system for stress evaluation based on EEG signal: A prospective review. In: Signal Processing and its Applications (CSPA), 2011 IEEE 7th International Colloquium on, 4-6 March 2011 2011, pp. 167-171

[33]. Lin, C.-J., Hsieh, M.-H.: Classification of mental task from EEG data using neural networks based on particle swarm optimization. Neurocomputing 72(4-6), 11211130 (2009). doi:http://dx.doi.org/10.1016/j.neucom.2008.0 2.017

[34]. Yazdani, A., Ebrahimi, T., Hoffmann, U.: Classification of EEG signals using Dempster Shafer theory and a k-nearest neighbor classifier. In: Neural Engineering, 2009. NER '09. 4th International IEEE/EMBS Conference on, April 29 2009-May 22009 2009, pp. 327330

[35]. Begum, S., Barua, S.: EEG sensor based classification for assessing psychological stress. Paper presented at the 10th International Conference on Wearable Micro and Nano Technologies for Personalized Health, Tallinn, June 26-28

[36]. Chin-Teng, L., Ruei-Cheng, W., Sheng-Fu, L., Wen-Hung, C., Yu-Jie, C., Tzyy-Ping, J.: EEG-based drowsiness estimation for safety driving using independent component analysis. Circuits and Systems I: Regular Papers, IEEE Transactions on 52(12), 2726-2738 (2005). doi:10.1109/TCSI.2005.857555

[37]. Yang, G., Lin, Y., Bhattacharya, P.: A driver fatigue recognition model using fusion of multiple features. In: Systems, Man and Cybernetics, 2005 IEEE International Conference on, 10-12 Oct. 2005 2005, pp. 1777-1784 Vol. 1772

[38]. Reisman, S.: Measurement of physiological stress. In: Bioengineering Conference, 1997., Proceedings of the IEEE 1997 23rd Northeast, 21-22 May 1997 1997, pp. 21-3

[39]. Ming, J., Zhelong, W.: A Method for Stress Detection Based on FCM Algorithm. In: Image and Signal Processing, 2009. CISP '09. 2nd International Congress on, 17-19 Oct. 2009 2009, pp. $1-5$

[40]. Begum, S., Ahmed, M., Funk, P., Xiong, N., Schéele, B.V.: Using Calibration and 
Fuzzification of Cases for Improved Diagnosis and Treatment of Stress. (2006)

[41]. Begum, S., Barua, S., Filla, R., Ahmed, M.U.: Classification of physiological signals for wheel loader operators using Multi-scale Entropy analysis and case-based reasoning. Expert Systems with Applications 41(2), 295$-305$ doi:http://dx.doi.org/10.1016/j.eswa.2013.05.0 68

[42]. Begum, S., Ahmed, M.U., Funk, P., Filla, R.: Mental state monitoring system for the professional drivers based on Heart Rate Variability analysis and Case-Based Reasoning. Paper presented at the Computer Science and Information Systems (FedCSIS), 2012 Federated Conference on, 9-12 Sept. 2012

[43]. Haak, M., Bos, S., Panic, S., Rothkrantz, L.J.M.: Detecting stress using eye blinks and brain activity from EEG signals. In: Proceeding of the 1st Driver Car Interaction, Jan 12008

[44]. Manjusha, M.A., Shermila, J.: Analysis of Automobile Drivers Stress By Using Physiological Signals. International Journal of Engineering Research and Applications (IJERA) (2014).

[45]. Sulaiman, N., Taib, M.N., Lias, S., Murat, Z.H., Aris, S.A.M., Mustafa, M., Rashid, N.A., Hamid, N.H.A.: Intelligent System for Assessing Human Stress Using EEG Signals and Psychoanalysis Tests. In: Computational Intelligence, Communication Systems and Networks (CICSyN), 2011 Third International Conference on, 26-28 July 2011 2011, pp. 363-367

[46]. Schmidt, E.A., Schrauf, M., Simon, M., Fritzsche, M., Buchner, A., Kincses, W.E.: Drivers' misjudgement of vigilance state during prolonged monotonous daytime driving. Accident Analysis \& Prevention 41(5), 1087-1093 (2009). doi:http://dx.doi.org/10.1016/j.aap.2009.06.00 7

[47]. Kar, S., Bhagat, M., Routray, A.: EEG signal analysis for the assessment and quantification of driver's fatigue. Transportation Research Part F: Traffic Psychology and Behaviour 13(5), 297-306 (2010). doi:http://dx.doi.org/10.1016/j.trf.2010.06.006

[48]. Schier, M.A.: Changes in EEG alpha power during simulated driving: a demonstration. International Journal of Psychophysiology 37(2), $155-162$ (2000). doi:http://dx.doi.org/10.1016/S01678760(00)00079-9
[49]. Lei, C., Jie, L., Yaoru, S., Huaping, Z., Chungang, Y.: EEG--- based vigilance analysis by using fisher score and PCA algorithm. In: Progress in Informatics and Computing (PIC), 2010 IEEE International Conference on, 10-12 Dec. 2010 2010, pp. 175-179

[50]. Lal, S.K.L., Craig, A., Boord, P., Kirkup, L., Nguyen, H.: Development of an algorithm for an EEG-based driver fatigue countermeasure. Journal of Safety Research 34(3), 321-328 (2003). doi:http://dx.doi.org/10.1016/S00224375(03)00027-6

[51]. Borghini, G., Astolfi, L., Vecchiato, G., Mattia, D., Babiloni, F.: Measuring neurophysiological signals in aircraft pilots and car drivers for the assessment of mental workload, fatigue and drowsiness.Neuroscience \& Biobehavioral Reviews(0) (2012). doi:http://dx.doi.org/10.1016/j.neubiorev.2012 .10 .003

[52]. Haak, P.v.d., Lon, R.v., Meer, J.v.d., Rothkrantz, L.: Stress assessment of cardrivers using EEG-analysis. Paper presented at the Proceedings of the 11th International Conference on Computer Systems and Technologies and Workshop for PhD Students in Computing on International Conference on Computer Systems and Technologies, Sofia, Bulgaria,

[53]. Balasubramanian, V., Adalarasu, K., Gupta, A.: EEG based analysis of cognitivefatigue during simulated driving. International Journal of Industrial and Systems Engineering 7(2), 135-149 doi:10.1504/IJISE.2011.038563

(2011).

[54]. Saidatul A., Paulraj Murugesa Pandiyan, Yaacob, S.: The Assessment of Developed Mental Stress Elicitation Protocol Based on Heart Rate and EEG Signals. International Journal of Computer Theory and Engineering 7(3) (2015). doi:10.7763/IJCTE.2015.V7.958

[55]. Calibo, T.K., Blanco, J.A., Firebaugh, S.L.: Cognitive stress recognition. In: Instrumentation and Measurement Technology Conference (I2MTC), $2013 \quad$ IEEE International, 6-9 May 2013 2013, pp. 14711475

[56]. Ha, U., Kim, C., Lee, Y., Kim, H., Roh, T., Yoo, H-.J.: A multimodal stress monitoring system with canonical correlation analysis. In: Engineering in Medicine and Biology Society (EMBC), 2015 37th Annual International Conference of the IEEE, 25-29 Aug. 2015 2015, pp. 1263-1266

[57]. Bin, H., Hong, P., Qinglin, Z., Bo, H., Majoe, D., Fang, Z., Moore, P.: Signal Quality 
Assessment Model for Wearable EEG Sensor on Prediction of Mental Stress. NanoBioscience, IEEE Transactions on 14(5), 553-561

(2015). doi:10.1109/TNB.2015.2420576

[58]. Chuang, C.-H., Huang, C.-S., Ko, L.-W., Lin, C.-T.: An EEG-based perceptual function integration network for application to drowsy driving. Knowledge-Based Systems 80, 143152 doi:http://dx.doi.org/10.1016/j.knosys.2015.01 .007

[59]. Awang, S.A., Paulraj, M.P., Yaacob, S.: Analysis of EEG signals by eigenvector methods. Paper presented at the Biomedical Engineering and Sciences (IECBES), 2012 IEEE EMBS Conference on, 17-19 Dec. 2012

[60]. Ardeenawatie, S., Pandiyan, P.M., Yaacob, S.: Mental Stress Level Classification Using Eigenvector Features and Principal Component Analysis. Communications in Information Science and Management Engineering 3(5), 254-261 (2013).

[61]. Aamodt, A., Plaza, E.: Case-based reasoning: foundational issues, methodological variations, and system approaches. AI Commun. 7(1), 39-59 (1994).

[62]. Lopez De Mantaras, R., Mcsherry, D., Bridge, D., Leake, D., Smyth, B., Craw, S., Faltings, B., Maher, M.L., Cox, M.T., Forbus, K., Keane, M., Aamodt, A., Watson, I.: Retrieval, reuse, revision and retention in case--- based reasoning. The Knowledge Engineering Review 20(03), 215--- 240 (2005). doi:doi:10.1017/S0269888906000646

[63]. Marling, C., Montani, S., Bichindaritz, I., Funk, P.: Synergistic case-based reasoning in medical domains. Expert Systems with Applications 41(2), 249-259 (2014). doi:http://dx.doi.org/10.1016/j.eswa.2013.05.0 63

[64]. Begum, S., Ahmed, M.U., Funk, P., Ning, X., Folke, M.: Case-Based Reasoning Systems in the Health Sciences: A Survey of Recent Trends and Developments. Systems, Man, and Cybernetics, Part C: Applications and Reviews, IEEE Transactions on 41(4), 421434

(2011). doi:10.1109/TSMCC.2010.2071862

[65]. Begum, S., Barua, S., Ahmed, M.: Physiological Sensor Signals Classification for Healthcare Using Sensor Data Fusion and Case-Based Reasoning. Sensors 14(7), 11770 (2014).

[66]. Begum, S., Ahmed, M., Funk, P., Xiong, N., vonSchéele, B.: Classify and Diagnose Individual Stress Using Calibration and Fuzzy Case-Based Reasoning. In: Weber, R.,
Richter, M. (eds.) Case-Based Reasoning Research and Development, vol. 4626. Lecture Notes in Computer Science, pp. 478491. Springer Berlin Heidelberg, (2007)

[67]. Harding, J., Walker, C., Walker, E.: Categories with fuzzy sets and relations. Fuzzy Sets and Systems(0). doi:http://dx.doi.org/10.1016/j.fss.2013.04.00 4

[68]. Jasper, H.H.: The ten twenty electrode system of the international federation. Electroencephalography and Clinical Neurophysiology 10, 371-375 (1958). doi:citeulike-article-id:9712821

[69]. Mammone, N., La Foresta, F., Morabito, F.C.: Automatic Artifact Rejection From Multichannel Scalp EEG by Wavelet ICA. Sensors Journal, IEEE12(3), 533-542 (2012). doi:10.1109/JSEN.2011.2115236

[70]. Castellanos, N.P., Makarov, V.A.: Recovering EEG brain signals: Artifact suppression with wavelet enhanced independent component analysis. Journal of Neuroscience Methods 158(2), 300-312 (2006) doi:http://dx.doi.org/10.1016/j.jneumeth.2006. 05.033

[71]. Akhtar, M.T., Mitsuhashi, W., James, C.J.: Employing spatially constrained ICAand wavelet denoising, for automatic removal of artifacts from multichannel EEG data. Signal Processing 92(2), 401-416 (2012). doi:http://dx.doi.org/10.1016/j.sigpro.2011.08. 005

[72]. Barua, S., Begum, S., Ahmed, M.U.: Intelligent Automated EEG Artifacts Handling Using Wavelet Transform, Independent Component Analysis and Hierarchal clustering, Workshop on Embedded Sensor Systems for Health through Internet of Things (ESS-H IoT) at 2nd EAI International Conference on IoT Technologies for HealthCare, 26-27 October

[73]. Mohammadi, S.: LYAPROSEN: MATLAB function to calculate Lyapunov exponent. Statistical Software Components (2009).

[74]. Übeyli, E.D.: Analysis of EEG signals by combining eigenvector methods and multiclass support vector machines. Computers in Biology and Medicine 38(1), 14-22 (2008). doi:http://dx.doi.org/10.1016/j.compbiomed.2 007.06.002

[75]. Wijayasekara, D., Manic, M.: Data driven fuzzy membership function generation for increased understandability. In: 2014 IEEE International Conference on Fuzzy Systems (FUZZ-IEEE), 6-11 July 2014 2014, pp. 133 140 
[76]. Ahmed, M.U., Begum, S., Funk, P., Xiong, N., von Scheele, B.: A multi-module casebased biofeedback system for stress treatment. Artificial Intelligence in Medicine 51(2), 107115 doi:http://dx.doi.org/10.1016/j.artmed.2010.09 .003

[77]. Ahmed, M.U., Loutfi, A.: Physical Activity Identification using Supervised Machine Learning and based on Pulse Rate. International Journal of Advanced Computer Science and Applications (IJACSA) 4(7) (2013).doi:http://dx.doi.org/10.14569/IJACSA .2013 .040730

[78]. Barua, S., Begum, S., Ahmed, M.U.: Supervised Machine Learning Algorithms to Diagnose Stress for Vehicle Drivers Based on Physiological Sensor Signals. Paper presented at the 12th International Conference on Wearable Micro and Nano Technologies for Personalized Health (pHealth 2015), Västerås, Sweden 